\title{
Application of Packed-nanofibers Solid-phase Extraction for Determination of Rhodamine B in Sausage
}

\author{
Lanlan Wei $^{1,2}$, Jianjun Deng ${ }^{3}$, Tao Kang ${ }^{4}$, Xuejun Kang*1,2 \\ 1.School of Public Health of Southeast University, Laboratory of Environment and Biosafety Research Institute \\ of Southeast University in Suzhou, Suzhou 215123, China \\ 2.Key Laboratory of Child Development and Learning Science (Ministry of Education), School of Biological \\ Science \& Medical Engineering, Southeast University, Nanjing 210096, China \\ 3. Suzhou Dongqi Biological Technology Co., LTD, Suzhou215123, China \\ 4. Jiangsu Dongda Jinzhi Information System co. LTD, Nanjing, 211100, China \\ E-mail:xjkang@163.com
}

Received: 14 November 2018; Accepted: 29 November 2018; Available online: 15 February 2019

\begin{abstract}
A method for the determination of Rhodamine B in sausage was developed and validated. After extraction of Rhodamine B with acetonitrile from foodstuffs, a novel electrospun polymer nanofibers packed micro-column was used for cleaning and concentrating of the analyte in the sample. High performance liquid chromatography with fluorescence detection (HPLC-Flu) was used for the determination of Rhodamine B in the sample. The mobile phase was composed of $3.0 \mathrm{~g} \mathrm{~L}^{-1}$ phosphate buffer and methanol (3:7, volume ratio), and the $\mathrm{pH}$ was adjusted to 7.0 with orthophosphoric acid. The results showed that the standard curve was linear over the validated concentrations range of 2-500 $\mathrm{ng} \mathrm{g}^{-1}$, and the limit of detection (LOD) and the limit of quantitation (LOQ) for Rhodamine B spiked samples was $0.2 \mathrm{ng} \mathrm{g}^{-1}$ and $0.7 \mathrm{ng} \mathrm{g}^{-1}$, respectively. The average recoveries of Rhodamine B were $90.4 \%-94.3 \%$ for sausage, and the relative standard deviation of the method was from $1.7 \%$ to $3.8 \%$. This proposed method was applied to real sample, and there was no Rhodamine B found in sausage.

Keywords: Sausage products; Packed-Fibers Solid-Phase extraction; High performance liquid chromatography; Rhodamine B; Electrospinning.
\end{abstract}

\section{Introduction}

Color is an important symbol of the food sensory quality. Therefore, people often use food coloring to improve the appearance of the food, making food enjoyable. Synthetic dyes offer good optical properties, stability and become the most economical food colorings. Currently, in China, the overuse and misuse of food additives have resulted in some food safety problems. Rhodamine B (RB) is an important water-soluble organic synthetic dye with carcinogenicity, teratogenicity, and mutagenicity. It is misused frequently and found in foodstuffs such as chili and red wine since they can preserve or restore the natural color of foodstuffs and enhance the appeal [1]. It is harmful if swallowed by human beings and animals and causes irritation to the skin, eyes, and respiratory tract [2]. China conducted a wide-ranging surveillance of the illegal use of synthetic organic dyes in foods from 2008, and RB is included in non-food substances by the Ministry of Health. Determination of RB in complex samples is challenging work because of a high level of interferences in foods when sausage is used as the sauce existing in them.

Accordingly, monitoring of Rhodamine B in high consumption food is necessary to ensure food safety. Several methods for the detection of Rhodamine B in food have been developed, with the common analytical methods including HPLC-UV [3, 4], HPLC-Flu [5-7], LC-PDA [8, 9], LC-MS [10], thin-layer chromatography [11] and spectophotometric flow-injection. Because high level of interferences bring difficulties for detection of Rhodamine $\mathrm{B}$ in foods, the pre-concentration separation methods including solid phase extraction [12], solvent extraction, gel chromatography purification and immunoaffinity chromatography ( IAC) column purification are widely used [13]. However, these methods often involve time consuming, solvent consuming and sample preparation steps, such as solvent extraction, concentration and cleanup, before chromatography analysis.

As far as we know, electrospinning is a versatile and straightforward method for producing thin fibers with diameters between micrometers and nanometers. Interestingly, when the diameters of polymer fiber materials decrease from micrometers to submicrons or nanometers, several surprising characteristics appear, such as very large surface area to volume ratio, the flexibility of surface functionality and the mechanical performance superior 
to any other known form of the material [14]. Owing to their unique mechanical and chemical properties, and being both cheap and easy to obtain, electrospun nanofibers have the potential to serve as a good sorbent material for SPE-based techniques. The main advantage of nanofiber materials is their large surface area to volume ratio that allows the use of a smaller sorbent bed mass, reducing the amount of organic solvents, required for rinsing and elution, and not needing a solvent evaporation for preconcentration of analytes. In this work, a PFSPE was used to separate and pre-concentrate the trace levels of Rhodamine B in sausage.

\section{Experimental}

\subsection{Reagents and solution}

Rhodamine B was obtained from the National Institute for the Control of Pharmaceutical and Biological Products (Beijing, China). Methanol (HPLC grade) and acetonitrile (HPLC grade) was obtained from the Shandong Yuwang Chemical Company (Shandong, China). Analytical grade disodium hydrogen phosphate and phosphate were purchased from Lingfeng Company (Shanghai, China). Polystyrene (PS, Mw = 185,000), dimethylformamide (DMF), tetrahydrofuran (THF). Standard stock solution was prepared at $0.1 \mathrm{mg} \mathrm{mL}^{-1}$ in pure water and stored at $4^{\circ} \mathrm{C}$ in the dark. The working solutions were prepared as appropriate dilution of the stock solutions.

\subsection{Equipment}

The HPLC system was an Shimadzu series (www.shimadzu.co.jp, Japan) equipped with an analytical column (SHIMADZU C18, $250 \mathrm{~mm} * 4.6 \mathrm{~mm}, 5 \mu \mathrm{m}$ ) attached to a fluorescence detector. Scanning electron microscopy (SEM) images were carried out on a Hitachi, S-3400 N microscope (www.hitachi.co.jp, Japan). Transmission electron microscopy (TEM) images were carried out on a JEM-2010 microscope (www.jeol.co. jp, Japan).

\subsection{Electrospinning}

Ten percent (w/v) PS solution was prepared by dissolving an appropriate amount PS in a mixture of dimethylformamide and tetrahydrofuran $(4: 6, \mathrm{v} / \mathrm{v})$. This solution was loaded into a glass syringe (5 mL volume). The glass syringe was fitted to a steel needle with a tip diameter of $0.5 \mathrm{~mm}$ whose tip was filed flat. A high-voltage generator was linked with the needle through a copper pin. A grounded iron drum mantled with a copper grid was served as the collection screen. The distance between the needle tip and the collector was $12 \mathrm{~cm}$. A voltage of 22 $\mathrm{kV}$ was supplied by a Dongwen high-voltage generator (Tianjin, China), model DW-P403-1AC. The flow rate of the syringe was controlled with a SLGO syringe pump (Beijing, China), model TCI-I. The feed rate of the precursor solution was fixed at $1.0 \mathrm{~mL} \mathrm{~h}^{-1}$.

\subsection{Method Development}

\subsubsection{Sample}

Sausage was purchased from a farmers' market in Nanjing, China. Typical samples without detectable Rhodamine B identified by a literature method were used as the blank samples [15]. The preparation procedure employed for spiked sausage sample entails the following steps: (1) $0.5 \mathrm{~g}$ crushed sausage was put into a $4 \mathrm{~mL}$ centrifuge tube; (2) added $100 \mu \mathrm{L}$ of Rhodamine B working solution; (3) added $2.0 \mathrm{~mL}$ of acetonitrile; (4) the mixture was sonicated for $10 \mathrm{~min}$ and centrifuged at $4000 \mathrm{r} \mathrm{min}^{-1}$ for $10 \mathrm{~min}$, the supernatant was separated; (5) the remaining residue was added $2 \mathrm{~mL}$ of acetonitrile to extract the target compound again; ( 6 ) two supernatants were combined and dried by nitrogen flow at room temperature; (7) the residue in the centrifuge tube was reconstituted in $100 \mu \mathrm{L}$ of ethanol and vortexing for $2 \mathrm{~min}$; (8) added $4 \mathrm{~mL}$ of $10 \%$ methanol water solution; (9) the mixture was centrifuged at $4000 \mathrm{r} \mathrm{min}^{-1}$ for $10 \mathrm{~min}$; (10) $2 \mathrm{~mL}$ of supernatant was transferred to a clean tube before the treatment by PFSPE (as shown in Fig.1).

\subsubsection{Chromatography condition}

The mobile phase was composed of an aqueous mobile phase $\left(3 \mathrm{~g} \mathrm{~L}^{-1}\right.$ phosphate buffer) and methanol (3:7, volume ratio), and adjusted $\mathrm{pH}$ to 7.0 with orthophosphoric acid. The HPLC flow rate was $1.0 \mathrm{~mL} \mathrm{~min}^{-1}$ and the injection volume was $20 \mu \mathrm{L}$. The optimum response of the fluorescent was observed with excitation and emission wavelengths set at $550 \mathrm{~nm}$ and $580 \mathrm{~nm}$, respectively. 


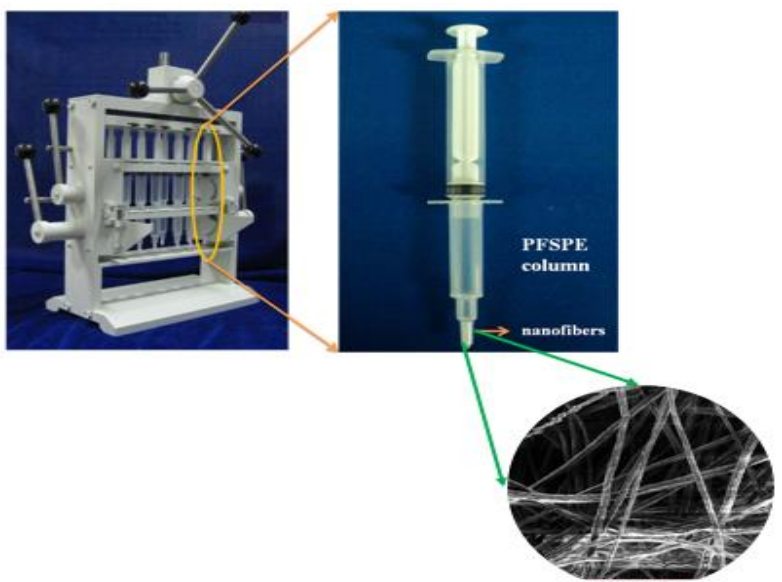

Fig. 1 Array SPE extraction device and PFSPE column

\section{Results and discussions}

\subsection{Characterization}

The PS nanofibers exhibited tiny nanofibers networks with uniform diameters of $750 \pm 110 \mathrm{~nm}$, it is clear that the surface morphology of composite nanofibers is rough. As observed from the images (Fig.2), the presence of the nanostructure could provide a high specific surface area and many interaction sites that could offer increased SPE efficiency. As shown in the TEM images of Fig.3, the surface of the nanofibers is smooth without obvious burrs.

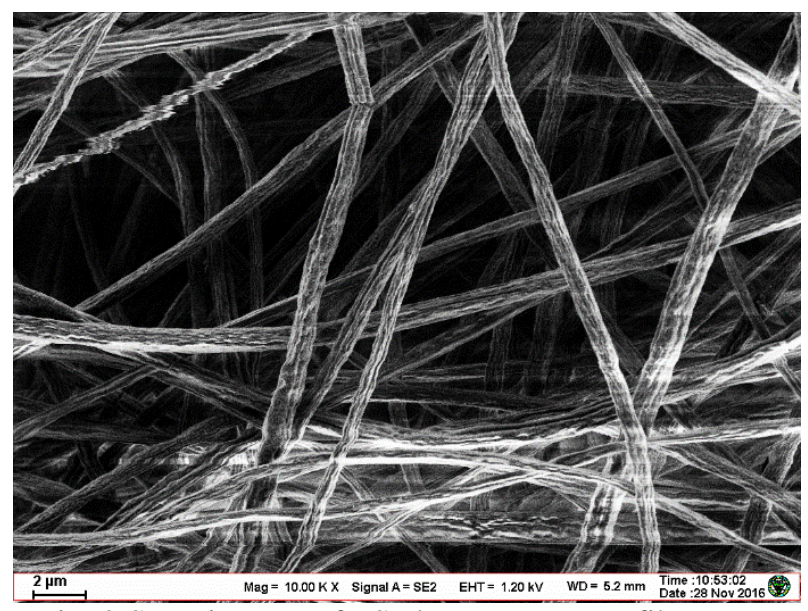

Fig. 2 SEM images of PS electrospun nanofibers

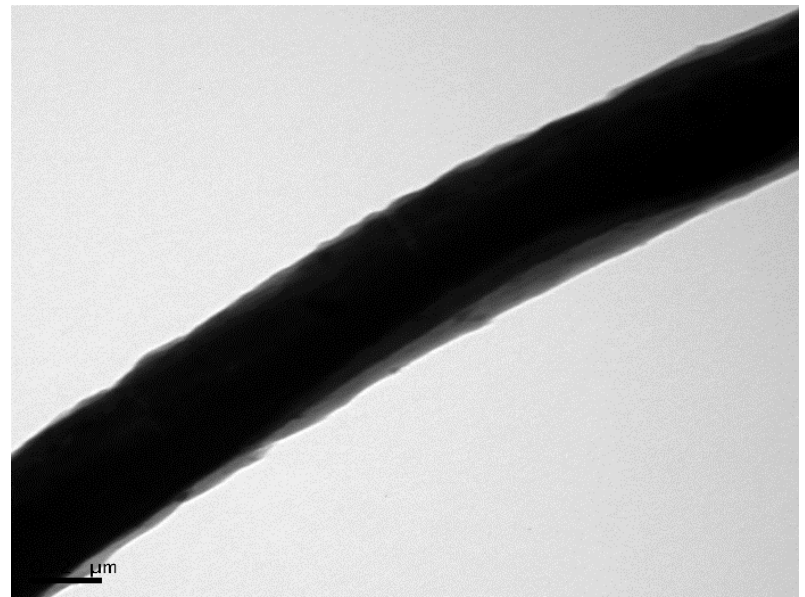

Fig. 3 TEM images of PS electrospun nanofibers 


\subsection{Validation of the method}

For the preparation of calibration curves, Rhodamine B was diluted at 2, 20, 80, 100, $500 \mathrm{ng} \mathrm{g}^{-1}$ in the blank samples of sausage. Each concentration point was operated parallel for three times going through the whole process of extraction-desorption determination, and the calibration curves are linear in the range of $2-500 \mathrm{ng} \mathrm{g}^{-1}$ for sausage with a good correlation $\left(\mathrm{R}^{2}=0.9944\right)$. The results are shown in Table 1.

Recoveries of the Rhodamine B were carried out in 3 replicates at 3 fortification levels $\left(10,50,200 \mathrm{ng} \mathrm{g}^{-1}\right)$ by spiking standard solution to $0.5 \mathrm{~g}$ of blank sausage. Good results are obtained at the three spiking levels, with recoveries in the range of $90.4 \%-94.3 \%$. The repeatability relative standard deviation (RSD) values of the method are ranged from $1.7 \%$ to $3.8 \%$ (as shown in Table 1). The limit of detection( LOD) and limit of quantification( LOQ) were determined by successive analyses of sample extracts with decreasing amounts of Rhodamine B until 3:1 and 10:1 signal-to-noise ratio were reached, respectively. In the blank sample matrix, the lowest assigned values obtained are reported in Table 1.

Table 1. Performance of the method

\begin{tabular}{|c|c|c|c|c|c|c|c|c|}
\hline \multirow{3}{*}{$\begin{array}{l}\text { Calibration } \\
\text { range } \\
\left(\mathrm{ng} \mathrm{g}^{-1}\right)\end{array}$} & \multicolumn{6}{|c|}{ Recovery $(\%) \pm \operatorname{RSD}(\mathrm{n}=3)$} & \multirow{3}{*}{$\begin{array}{l}\text { LOD } \\
\left(n g g^{-1}\right)\end{array}$} & \multirow{3}{*}{$\begin{array}{l}\text { LOQ } \\
\quad\left(n g g^{-1}\right)\end{array}$} \\
\hline & & & & \multirow{2}{*}{\multicolumn{3}{|c|}{ Between-day }} & & \\
\hline & \multicolumn{3}{|c|}{ Spiked concentration $\left(\mathrm{ng} \mathrm{mL} \mathrm{m}^{-1}\right)$} & & & & & \\
\hline \multirow{2}{*}{$2-500$} & 10 & 50 & 200 & 10 & 50 & 200 & \multirow{2}{*}{0.2} & \multirow{2}{*}{0.7} \\
\hline & $90.4 \pm 2.6$ & $92.7 \pm 2.1$ & $95.8 \pm 1.7$ & $88.9 \pm 3.8$ & $91.5 \pm 3.4$ & $94.3 \pm 2.8$ & & \\
\hline
\end{tabular}

\subsection{Determination of real samples}

To further demonstrate the effect of the PFSPE pre-treatment method, anonymous sample from local markets (Nanjing, China) was subjected to extraction using the PFSPE process and analysis via the proposed procedure. The sample was then spiked with Rhodamine B standards at $100 \mathrm{ng} \mathrm{g}^{-1}$. Rhodamine B was detected in sausage sample (as shown in Figure 4).

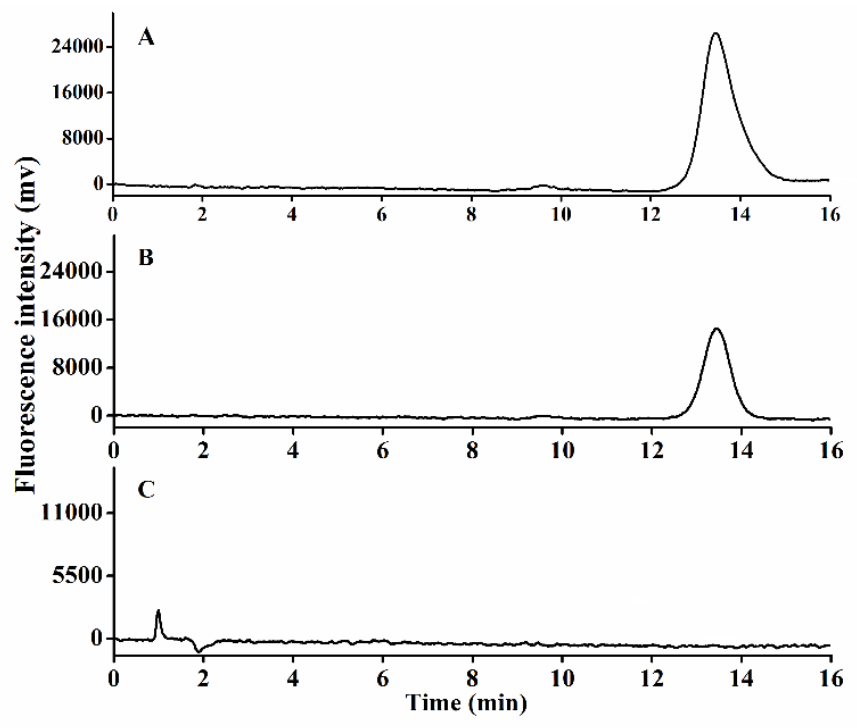

Fig. 4 HPLC-FLU spectra of real samples. (A) Chromatogram of standard solution after extraction; (B) chromatogram of sausage sample spiked with Rhodamine B after extraction and (C) chromatogram of sausage sample after extraction.

\section{Conclusions}

A fast, sensitive HPLC-FLU method for the determination of Rhodamine B in sausage was presented in this paper. This method possessed high sensitivity and selectivity to ensure accurate measurement of Rhodamine B in the food. The PFSPE pretreatment method offered simple, fast and low-cost operation. The microlitre amount of eluting solvent can improve the conventional enrichment process, which obviously illustrated the environmental 
friendliness. Moreover, higher extraction recoveries and good reproducibility were especially suitable for trace analysis of the samples.

\section{Acknowledgments}

This study was supported by the National Science Foundation of China (No. 81673230); the Social Development Research Program of Jiangsu Province Science and Technology department (No. BE2016741); and the Postgraduate Research \& Practice Innovation Program of Jiangsu Province (No.KYCX17_0189).

\section{Reference}

[1] Yu J S, Zhou T Y. The electrochemistry and thin-layer luminescence spectroelectrochemistry of Rhodamine $6 \mathrm{G}$ at a 4, 4'-bipyridine-modified gold electrode. Journal of Electroanalytical Chemistry.2001; 504(1):89-95.

[2] Jain R, Mathur M, Sikarwar S, et al. Removal of the hazardous dye Rhodamine B through photocatalytic and adsorption treatments. Journal of Environmental Management. 2007; 85(4):956-964.

[3] Xiao-Yan, LI, Mei LI, Chen Q F, et al. Determination of Rhodamine B in red wine by solid phase extractionhigh performance liquid chromatography. Food Science. 2011; 32(8):238-243.

[4] Lei-long, SUN, Zhi-hua Y. HPLC determination of Rhodamine B in foods. Chinese Journal of Health Laboratory Technology. 2011; 7: 023.

[5] Lu M, Zhen-Hua G, Jia-Lin M O, et al. Determination of Rhodamine B in condiment by ultra performance liquid chromatography with fluorescence detector. Guangzhou Chemistry. 2011.

[6] HU Xia, XIAO Guang, PAN Wei, et al. Simultaneous determination of 7 Rhodamine dyes in hot chili products by high performance liquid chromatography-tandem mass spectrometry. Chromatographic. 2010; 28(6):590-595.

[7] Hui C, Bing LI, Chun-Rui Z. HPLC-MS/MS Determination of Rhodamine B residue in Chinese preserved sausages. Food Science. 2010; 31(4):223-225.

[8] Li-Ya X, Yuan-Yuan H , Lin-He K, et al. Simultaneous determination of basic orange2, metanil yellow, tartrazine, sunset yellow in bean-product and acid orange II, ponceau 2R, Rhodanmine B in chili powder by thin-layer chromatographic scanning. Chinese Journal of Analysis Laboratory. 2010.

[9] Soylak M, Unsal Y E, Yilmaz E, et al. Determination of Rhodamine B in soft drink, waste water and lipstick samples after solid phase extraction. Food and chemical toxicology. 2011; 49(8): 1796-1799.

[10] Amate C F, Unterluggauer H, Fischer R J, et al. Development and validation of a LC-MS/MS method for the simultaneous determination of aflatoxins, dyes and pesticides in spices. Analytical and bioanalytical chemistry. 2010;397(1): 93-107.

[11] Acar O, Özvatan S, Ilim M. Determination of cadmium, copper, iron, manganese, lead and zinc in lichens and botanic samples by electrothermal and flame atomic absorption spectrometry. Turkish Journal of Chemistry. 2005; 29(4):335-344.

[12] Mudabuka B, Ondigo D, Degni S, et al. A colorimetric probe for ascorbic acid based on copper-gold nanoparticles in electrospun nylon. Microchimica Acta. 2014; 181(3-4):395-401.

[13] Chen J, Teo KC. Determination of cadmium, copper, lead and zinc in water samples by flame atomic absorption spectrometry after cloud point extraction. Anal Chim Acta. 2001; 450:215-222.

[14] Mudabuka B, Ondigo D, Degni S, Vilakazi S, Torto N. A colorimetric probe for ascorbic acid based on copper-gold nanoparticles in electrospun nylon. Microchim Acta. 2014; 181:395-401.

[15] Zhao R, Chu L, Wang Y, et al. Application of packed-fiber solid-phase extraction coupled with GC-MS for the determination of short-chain fatty acids in children's urine. Clinica Chimica Acta, 2017; 468:120-125.

(C) 2019 by the author(s). This work is licensed under a Creative Commons Attribution 4.0 International License (http://creativecommons.org/licenses/by/4.0/). Authors retain copyright of their work, with first publication rights granted to Tech Reviews Ltd. 